\title{
AVALIAÇÃO MULTIDIMENSIONAL DA MULHER IDOSA EM UM CENTRO DE CONVIVÊNCIA
}

\section{MULTIDIMENSIONAL EVALUATION OF ELDERLY WOMEN IN A}

\section{Monica Cavalcante ${ }^{1} *$ Larissa Silva Santos $^{2} *$ Edna Silva Cantanhede $^{3} *$ Tatiane Barbosa de $^{2}$ Lira $^{4} *$ Tércia Carine Silva Leite ${ }^{5} *$ Jardel Alves Pereira de Freitas $^{6} *$ Elian da Silva Jorge $^{7 *}$ Francisca Cecília Viana Rocha ${ }^{8}$}

\section{RESUMO}

Objetivo: Analisar por meio da avaliação multidimensional os determinantes do envelhecimento ativo em Mulheres Idosas. Métodos: Trata-se de uma pesquisa descritiva, com abordagem quantitativa realizada em um Centro de Convivência, de caráter público, situado na cidade de Teresina, Piauí, Brasil. Os participantes do estudo foram 76 mulheres idosas que frequentem o Centro de Convivência. A coleta dos dados ocorreu no Centro de Convivência por meio da aplicação de um questionário nos meses de outubro e novembro de 2018. Os dados foram registrados em uma planilha Microsoft Excel e posteriormente exportadas no programa IbmSpssStatistics 20.0 para tabulação dos dados. Resultados: Ao analisar os dados sócio demográficos observou-se que a idade média foi entre 60 a 64 anos, sendo que 36,84\% das idosas conviviam com cônjuges ou companheiros, a maioria das idosas $(56,58 \%)$ recebem entre um e três salários mínimos por mês. A comorbidade que teve o maior índice foi o sobrepeso com $25,18 \%$ e a queda atingindo 49,68\%. Os determinantes do envelhecimento ativo 40,79\% das idosas tinham dificuldades com a visão e cerca de $84,21 \%$ não tem escada no domicílio e $56 \%$ das idosas usam tapete solto $(56,58)$. Conclusão: Portanto, apesar que nem todas as idosas obtiveram uma avaliação positiva em cada domínio da avaliação multidimensional, em uma visão geral apresentaram com envelhecimento ativo.

Palavras-chave: Enfermagem Geriátrica; Idosa; Envelhecimento Ativo; Saúde da Mulher.

\section{ABSTRACT}

Objective: To analyze, through multidimensional evaluation, the determinants of active aging in Elderly Women. Methods: This is a descriptive study, with a quantitative approach, carried out in a Public Health Center, located in the city of Teresina, Piauí, Brazil. The study participants were 76 elderly women who attend the Community Center. Data collection took place at the Community Center through the application of a questionnaire in the months of October and November 2018. The data were recorded in a Microsoft Excel spreadsheet and later exported in the IbmSpssStatistics 20.0 program for data tabulation. Results: When analyzing the socio-demographic data, it was observed that the average age was between 60 and 64 years, with 36,84\% of the elderly women living with spouses or partners, most elderly women $(56,58 \%)$ receive between one and three minimum wages per month. The comorbidity that had the highest rate was overweight with $25,18 \%$ and the fall reaching 49,68\%. The determinants of active aging 40,79\% of the elderly women had difficulties with their vision and about $84,21 \%$ have no stairs at home and $56 \%$ of the elderly women use a loose carpet $(56,58)$. Conclusion: Therefore, although not all elderly women obtained a positive evaluation in each domain of multidimensional evaluation, in an overview they presented with active aging.

Keywords: Geriatric Nursing; Elderly; Active Anging; Women's Health

\footnotetext{
${ }^{1}$ Enfermeira. Especialista em Unidade de Terapia Intensiva,Uniredentor/AMIB, Teresina, Piauí. Brasil.

${ }^{2}$ Enfermeira. Graduada em Enfermagem pela UNINOVAFAPI, Teresina, Piauí. Brasil.

${ }^{3}$ Enfermeira. Graduada em Enfermagem pela UNINOVAFAPI, Teresina, Piauí. Brasil.

${ }^{4}$ Enfermeira. Residente em Cuidados Intensivos pelo Hospital Universitário da Universidade Federal do Piauí, Teresina-PI. Brasil.

${ }^{5}$ Enfermeira. Graduada em Enfermagem pela UNINOVAFAPI, Teresina, Piauí. Brasil.

${ }^{6}$ Enfermeiro. Graduada em Enfermagem pela UNINOVAFAPI, Teresina, Piauí. Brasil.

${ }^{7}$ Enfermeira. Graduada em Enfermagem pela UNINOVAFAPI, Teresina, Piauí. Brasil.

${ }^{8}$ Enfermeira. Mestre em Enfermagem. Docente do Departamento de Enfermagem do Centro Universitário UNIOVAFAPI. Teresina, PI, Brasil.

https://doi.org/10.31011/reaid-2021-v.95-n.36-art.1085 Rev Enferm Atual In Derme v. 95, n. 36, 2021 e-021143
} 


\section{INTRODUÇÃO}

O envelhecimento saudável é definido por medidas subjetivas, tais como: satisfação de vida, afetos e disposição de espírito, e por medidas objetivas, nomeadamente, morbidade, independência e mortalidade. A partir de uma perspectiva biomédica, a definição mais proeminente descreve-o em termos de três critérios: baixo risco para doenças e deficiências relacionadas à doença, alta atividade mental e física e envolvimento ativo na vida cotidiana ${ }^{(1)}$.

\section{A Avaliação Multidimensional do} Idoso permite o reconhecimento das demandas biopsicossociais do indivíduo, ou seja, o diagnóstico de suas condições de saúde agudas e/ou crônicas. Este diagnóstico clínico-funcional deve ser capaz de reconhecer as incapacidades, tanto no que se refere à independência e autonomia nas atividades de vida diária (funcionalidade global), quanto à presença de comprometimento dos sistemas funcionais principais, representados pela cognição, humor, mobilidade e comunicação ${ }^{(2)}$.

$\mathrm{O}$ entendimento dos determinantes e dos fatores etiológicos do envelhecimento saudável - sejam eles relacionados às condições e desigualdades socioeconômicas, características individuais (biológicas, psicológicas, genéticas) e comportamentais, ambiente físico, acesso e utilização de serviços de saúde, ademais das diferenças culturais e de gênero - tornam-se fundamentais para subsidiar o planejamento de políticas de atenção à saúde da população idosa ${ }^{(3)}$.

O aumento da expectativa de vida traz novos contornos à população mundial. No que diz respeito ao Brasil, a Organização Mundial da Saúde estima que, até 2025, esse será o sexto país no mundo com maior número de idosos, alcançando 30 milhões de pessoas. Quanto à população feminina, a estatística mostra que é mais numerosa que a masculina ${ }^{(4)}$.

A principal característica do envelhecimento saudável é a capacidade de aceitar as mudanças fisiológicas decorrentes da idade, sendo que as doenças e limitações não impossibilitam a experiência pessoal de velhice bem-sucedida. O bem-estar subjetivo é um critério essencial para a velhice bemsucedida, aproxima-se de um princípio organizacional para alcance de metas, expandindo-se em um continuo multidimensional, que ultrapassa a objetividade da saúde física ${ }^{(5)}$.

Neste sentido, tem vindo a ser cada vez mais debatida e defendida pelos especialistas a realização de uma avaliação multidimensional dos idosos, cujas características individuais e particularidades biopsicossociais são essenciais no correto diagnóstico da sua condição geral, permitindo assim definir corretamente o plano de intervenção junto do utente, seja de reabilitação, prevenção ou educação ${ }^{(6)}$. 
Nesse processo de envelhecimento a mulher passa pelo climatério, um período transicional, polêmico e crítico. Fase biológica da mulher, compreendendo a transição entre o período reprodutivo e o não reprodutivo, com início por volta dos 35 anos e encerrando-se aos 65 anos. Abrange a menopausa, correspondendo ao último ciclo menstrual, reconhecido depois de passados 12 meses da sua ocorrência, o que, em geral, se dá por volta dos 48 aos 50 anos de idade ${ }^{(7)}$.

Os grupos de convivência têm sido uma alternativa estimulada em todo o Brasil. De maneira geral, inicialmente os idosos buscam, nesses grupos, melhoria física $\mathrm{e}$ mental, por meio de exercícios físicos. Posteriormente, as necessidades aumentam, e as atividades de lazer, como viagens, também ganham espaço, além do desenvolvimento de outras atividades, sempre promovendo atividades ocupacionais e lúdicas ${ }^{(5)}$.

Portanto, o envelhecimento da população é algo crescente mundialmente e deve se salientar que o processo de envelhecimento saudável e ativo é algo resultante de um conjunto de fatores que promovem uma melhor qualidade de vida para os idosos. A partir da problemática da pesquisa o objetivo do estudo é a Avaliação multidimensional da Mulher Idosa em um Centro de Convivência. Neste contexto, a seguinte hipótese norteou o desenvolvimento do estudo: Qual a avaliação multidimensional dos determinantes do envelhecimento ativo em mulheres idosas?

\section{MÉTODOS}

Trata-se de uma pesquisa descritiva com abordagem quantitativa. A pesquisa descritiva visa descrever as características de determinadas populações ou fenômenos. Uma de suas peculiaridades está na utilização de técnicas padronizadas de coleta de dados, tais como o questionário e a observação sistemática $^{(8)}$. A pesquisa quantitativa reúne evidências empíricas e são números que resultam de algum tipo de medição formal que são analisados através de procedimentos estatísticos. Ressalta também que esse tipo de pesquisa não deve ser usado para responder questão ética ou moral ${ }^{(9)}$.

O cenário da pesquisa foi um Centro de Convivência, de caráter público, onde são desenvolvidas atividades com: artesanato, canto e coral, educação física e orientação sexual sócio pedagógica com 94 idosas. Participaram do estudo mulheres idosas que frequentem o Centro de Convivência. O cálculo da amostra para uma população finita de 94 idosas $\left(\mathrm{N}^{\circ}\right)$ com margem de erro (E) de $5 \%$ e nível de confiança de $95 \%(\mathrm{z}=1.96)$ e $\mathrm{P}=0.50$ (parâmetro). Assim seguido o cálculo chegou a uma amostra de 76 idosas.

Foram incluídas idosas que frequentem o Centro de Convivência há pelo menos seis meses, com idade a partir de 60 anos e que concordassem em participar da 
pesquisa por meio da assinatura do Termo de Consentimento Livre e Esclarecido (TCLE). Excluiu-se idosas que entraram no Centro de Convivência por um período inferior há 6 meses e com idade inferior a 60 anos, idosas com cognição não preservada e que apresentem discordância na assinatura do TCLE. Para identificar a cognição das idosas foi realizado o MEEM (Mini Exame do Estado Mental).

A coleta de dados foi realizada após aprovação pelo Comitê e Pesquisa do Centro Universitário UNINOVAFAPI e das instituições co-participantes. Após o esclarecimento sobre os objetivos e metodologia da pesquisa, com consequente assinatura do Termo de Consentimento Livre e Esclarecido (TCLE), os dados da pesquisa foram coletados diretamente com as idosas do centro de convivência por meio da aplicação de um questionário.

O questionário é um instrumento validado pelo Ministério da Saúde o mesmo faz uma síntese da estrutura da avaliação multidimensional da pessoa idosa. Representa uma avaliação rápida que pode ser utilizado para identificar problemas de saúde condicionantes de declínio funcional em pessoas idosas.

O questionário foi aplicado individualmente nos meses de outubro e novembro de 2018, em uma sala do Centro de Convivência, com porta fechada, boa iluminação e com a presença apenas dos pesquisadores, favorecendo o conforto $\mathrm{e}$ tranquilidade aos participantes, bem como preservando a sua identidade. Ressalta-se que a data da aplicação do questionário foi previamente agendada, respeitando a disponibilidade de tempo dos participantes e visando não inferir nas suas atividades. $\mathrm{O}$ tempo de duração ocorreu em torno de trinta minutos.

Os questionários após aplicados foram registrados na planilha Microsoft Excel e posteriormente exportadas para o programa IBM SPSS STATISTICS 20.0 que fará a tabulação dos dados. Análise estatística foi do tipo descritiva, as variáveis categóricas analisadas por meio da leitura das frequências absolutas $\left(\mathrm{N}^{\circ}\right)$ e relativos $(\%)$. As variáveis quantitativas tiveram analise pela leitura das estatísticas de posição (Média) e de variabilidade (desvio padrão). O teste de associação do qui-quadrado será usado com nível de significância de 5\% os resultados estarão expostos em forma de tabela e gráficos.

A presente pesquisa obedeceu à Resolução 466/2012 do Conselho Nacional de Saúde, que trata de pesquisas com seres humanos. O projeto foi aprovado pelo Comitê de Ética e Pesquisa (CEP) do Centro Universitário UNINOVAFAPI com o CAAE $n^{\circ}$ 2.819.798, bem como pela instituição Coparticipante. A referida Resolução incorpora, sob a ótica do indivíduo e das coletividades, referenciais da bioética, tais como, autonomia, 
não maleficência, beneficência, justiça e equidade, dentre outros, e visa a assegurar os direitos e deveres que dizem respeito aos participantes da pesquisa, à comunidade científica e ao Estado ${ }^{(10)}$.

Os participantes da pesquisa foram esclarecidos sobre o estudo e seus objetivos. A cada participante foi entregue o TCLE, que garante a confidência, a privacidade e a proteção de imagem dos entrevistados, onde estes estarão totalmente isentos de qualquer custo e livres a desistir ao qualquer momento de participar durante a coleta de dados

\section{RESULTADOS}

Ao analisar os dados socio demográficos, expressos na tabela 1 , foi possível observar que a idade média das entrevistadas foi de 60 a 64 anos, a maioria das idosas pesquisadas $(47,37 \%)$ possuíam até o ensino fundamental. Observou-se que $36,84 \%$ das idosas conviviam com cônjuges ou companheiros, $34,21 \%$ eram viúvos e $21,05 \%$, solteiros. Quanto a renda familiar, a maioria das idosas $(56,58 \%)$ recebe entre um e três salários mínimos por mês.

Tabela 1 - Características sociodemográficas das

Idosas. Teresina (PI), novembro 2018 a abril 2019

\begin{tabular}{ccc}
\hline \hline \multicolumn{1}{l}{ Características } & $\mathrm{N}^{\mathbf{0}}$ & $\%$ \\
\hline 60 a 64 & $\mathbf{2 7}$ & $\mathbf{3 5 , 5 3}$ \\
65 a 69 & $\mathbf{1 8}$ & $\mathbf{2 3 , 6 8}$ \\
70 a 74 & $\mathbf{1 2}$ & $\mathbf{1 5 , 7 9}$
\end{tabular}

Faixa etária

$\begin{array}{ccc}75 \text { a } 79 & \mathbf{1 3} & \mathbf{1 7 , 1 1} \\ 80 \text { a } 85 & \mathbf{6} & \mathbf{7 , 8 9} \\ \text { Total } & \mathbf{7 6} & \mathbf{1 0 0 , 0 0}\end{array}$

$\begin{array}{lll}\text { Sem } & \mathbf{6} & \mathbf{7 , 8 9}\end{array}$

Escolaridade Só lê e escreve $\quad \mathbf{8} \quad \mathbf{1 0 , 5 3}$

Até ensino

fundamental 


\begin{tabular}{|c|c|c|c|}
\hline \multicolumn{2}{|r|}{$\begin{array}{l}\text { Até ensino } \\
\text { médio }\end{array}$} & 12 & 15,79 \\
\hline & $\begin{array}{c}\text { Superior } \\
\text { incompleto }\end{array}$ & - & - \\
\hline & Superior & 14 & 18,42 \\
\hline & Total & 76 & 100,00 \\
\hline & Solteira & 16 & 21,05 \\
\hline & Casada & 28 & 36,84 \\
\hline & Viúva & 26 & 34,21 \\
\hline Estado civil & Divorciada & 5 & 6,58 \\
\hline & União estável & - & - \\
\hline & Outras & 1 & 1,32 \\
\hline & Total & 76 & 100,00 \\
\hline
\end{tabular}

Menos de R\$ 954,00 18 23,68

$\mathrm{R} \$ 954,00$ a $\mathrm{R} \$$

$$
1.908,00
$$
56,58

Renda familiar $\mathrm{R} \$ 1.908,00 \mathrm{a}$ $\mathrm{R} \$ 4.770,00$

Mais de R\$

$4.770,00$

$5 \quad 6,58$

$\begin{array}{lll}\text { Total } & \mathbf{7 6} & \mathbf{1 0 0 , 0 0}\end{array}$

Fonte: Pesquisa direta

De acordo com análise da tabela 2 a maioria das idosas não apresentavam comorbidades, mostrando-se satisfeita com a vida, realizando as atividades propostas no questionário $\mathrm{A}$ comorbidade que teve o maior índice foi o sobrepeso com $25,18 \%$ e a queda atingindo 
49,68\%. Apesar da presença de idosos dessa amostra, porém mostraram-se comorbidades, muito frequente entre os satisfeita com a vida.

Tabela 2 - Comorbidades nas mulheres idosas. Teresina (PI), novembro 2018 a abril 2019

\begin{tabular}{llcr}
\hline \hline Comorbidades & & $\mathrm{N}^{\circ}$ & $\%$ \\
\hline \multirow{2}{*}{$\begin{array}{l}\text { Presença de } \\
\text { incontinência }\end{array}$} & Não & $\mathbf{3}$ & $\mathbf{3 , 9 5}$ \\
& Total & $\mathbf{7 3}$ & $\mathbf{9 6 , 0 5}$ \\
& & $\mathbf{7 6}$ & $\mathbf{1 0 0 , 0 0}$
\end{tabular}

Frequência da incontinência

\begin{tabular}{|c|c|c|c|}
\hline & $\begin{array}{l}\text { Não tem } \\
\text { incontinência }\end{array}$ & 73 & 96,05 \\
\hline $\begin{array}{l}\text { Incontinência provoca } \\
\text { incômodo ou }\end{array}$ & Sim & 3 & 3,95 \\
\hline embaraço & Não & & \\
\hline & Total & 76 & 100,00 \\
\hline
\end{tabular}

IMC

25,1

8

$\begin{array}{llrr} & \text { Sim } & \mathbf{7 1} & \mathbf{9 3 , 4 2} \\ \text { Função dos MMSS } & \text { Não } & \mathbf{5} & \mathbf{6 , 5 8} \\ & \text { Total } & \mathbf{7 6} & \mathbf{1 0 0 , 0 0} \\ & \text { Sim } & \mathbf{7 4} & \mathbf{9 7 , 3 7} \\ \text { Função dos MMII } & \text { Não } & \mathbf{2} & \mathbf{2 , 6 3} \\ & \text { Total } & \mathbf{7 6} & \mathbf{1 0 0 , 0 0} \\ \text { Se sente triste ou } & \text { Sim } & \mathbf{2 5} & \mathbf{3 2 , 8 9}\end{array}$




$\begin{array}{llcr}\text { desanimada } & \text { Não } & \mathbf{5 1} & \mathbf{6 7 , 1 1} \\ \text { frequentemente } & \text { Total } & \mathbf{7 6} & \mathbf{1 0 0 , 0 0} \\ & \text { Sim } & \mathbf{3 9} & \mathbf{5 1 , 3 2} \\ & \text { Não } & \mathbf{3 7} & \mathbf{4 8 , 6 8} \\ \text { Queda } & \text { Total } & \mathbf{7 6} & \mathbf{1 0 0 , 0 0}\end{array}$

Frequência de queda

Fonte: Pesquisa direta

Em relação aos determinantes do necessita da visão, a dificuldade é evidente envelhecimento ativo das mulheres idosas devido a idade. Os demais determinantes a pesquisadas na tabela 3 demonstra que cerca maioria das idosas realizavam sem de $40,79 \%$ das idosas tinham dificuldades em dificuldades.

dirigir, ver TV ou outras atividades que

Tabela 3 - Avaliação da capacidade funcional. Teresina (PI),

Novembro 2018 a Abril 2019

\begin{tabular}{llcc}
\hline \hline Determinantes & & $\mathrm{N}^{\circ}$ & $\%$ \\
\hline $\begin{array}{l}\text { Dificuldade visuais em } \\
\text { dirigir, ver TV ou outra }\end{array}$ & Não & $\mathbf{3 1}$ & $\mathbf{4 0 , 7 9}$ \\
atividade diária & Total & $\mathbf{7 6}$ & $\mathbf{5 9 , 2 1}$ \\
& Sim & $\mathbf{6 4}$ & $\mathbf{1 0 0 , 0 0}$ \\
$\begin{array}{l}\text { Responde à pergunta } \\
\text { feita - teste do sussurro }\end{array}$ & Não & $\mathbf{1 2}$ & $\mathbf{8 4 , 2 1}$ \\
& Total & $\mathbf{7 6}$ & $\mathbf{1 5 , 7 9}$ \\
Problema em desfrutar & Sim & $\mathbf{5}$ & $\mathbf{1 0 0 , 0 0}$ \\
prazer nas relações & Não & $\mathbf{7 1}$ & $\mathbf{6 , 5 8}$ \\
sexuais & Total & $\mathbf{7 6}$ & $\mathbf{9 3 , 4 2}$
\end{tabular}




\begin{tabular}{|c|c|c|c|}
\hline Consegue repetir as & Sim & 52 & 68,42 \\
\hline \multirow{2}{*}{$\begin{array}{l}\text { palavras - cognição e } \\
\text { memória }\end{array}$} & Não & 24 & 31,58 \\
\hline & Total & 76 & 100,00 \\
\hline \multirow{3}{*}{$\begin{array}{l}\text { Atividades diárias - sair } \\
\text { da cama }\end{array}$} & Sim & 76 & 100,00 \\
\hline & Não & - & - \\
\hline & Total & 76 & 100,00 \\
\hline \multirow{3}{*}{$\begin{array}{l}\text { Atividades diárias - } \\
\text { vestir-se }\end{array}$} & Sim & 76 & 100,00 \\
\hline & Não & - & - \\
\hline & Total & 76 & 100,00 \\
\hline \multirow{3}{*}{$\begin{array}{l}\text { Atividades diárias - } \\
\text { preparar sua refeições }\end{array}$} & Sim & 72 & 94,74 \\
\hline & Não & 4 & 5,26 \\
\hline & Total & 76 & 100,00 \\
\hline \multirow{3}{*}{$\begin{array}{l}\text { Atividades diárias - } \\
\text { fazer compras }\end{array}$} & Sim & 70 & 92,11 \\
\hline & Não & 6 & $\mathbf{7 , 8 9}$ \\
\hline & Total & 76 & 100,00 \\
\hline
\end{tabular}

Fonte: Pesquisa direta

Conforme se verifica na tabela 4 cerca de $84,21 \%$ não tem escada no domicilio e $56 \%$ das idosas usam tapete solto $(56,58)$ e $88,16 \%$ não tem corrimão no banheiro do domicílio fator importante, pois é um risco para a segurança e o envelhecimento saudável. Sabe- se que esses equipamentos de proteção ajudam para evitar que os idosos sofram acidentes, principalmente a queda um fator que causa na maioria das vezes fraturas de ossos.

Tabela 4 - Dificuldades para manter o envelhecimento ativo e saudável nas mulheres idosas. Teresina (PI), novembro 2018 a abril 2019 


\begin{tabular}{|c|c|c|c|c|}
\hline Dificuldades & & $\mathrm{N}^{\circ}$ & $\%$ & \\
\hline \multirow{3}{*}{$\begin{array}{l}\text { Tem escada no } \\
\text { domicílio }\end{array}$} & Sim & 12 & 15,79 & \\
\hline & Não & 64 & 84,21 & \\
\hline & Total & 76 & 100,00 & \\
\hline \multirow{3}{*}{$\begin{array}{l}\text { Tem tapete } \\
\text { soltos no } \\
\text { domicílio }\end{array}$} & Sim & 43 & 56,58 & \\
\hline & Não & 33 & 43,42 & \\
\hline & Total & 76 & 100,00 & \\
\hline \multirow{3}{*}{$\begin{array}{l}\text { Tem corrimão } \\
\text { no banheiro do } \\
\text { domicílio }\end{array}$} & Sim & 9 & 11,84 & \\
\hline & Não & 67 & 88,16 & \\
\hline & Total & 76 & 100,00 & \\
\hline \multirow{3}{*}{$\begin{array}{l}\text { Alguém } \\
\text { ajudaria em } \\
\text { caso de doença } \\
\text { ou } \\
\text { incapacidade }\end{array}$} & Sim & 72 & 94,74 & \\
\hline & Não & 4 & & 5,26 \\
\hline & Total & 76 & & 100,00 \\
\hline
\end{tabular}

Fonte: Pesquisa direta

\section{DISCUSSÃO}

Os principais achados desse estudo mostraram que essas idosas são ativas e com uma qualidade de vida satisfatória. Os aspectos mais prevalentes sociodemográficos evidenciaram que a idosa avaliada caracterizava-se de uma população com estado civil casado, com renda de uma a dois salários mínimos e com escolaridade até o ensino fundamental.
Todos os fatores determinantes do envelhecimento ativo têm em comum a convergência na cultura e no gênero, que são considerados determinantes transversais. A cultura abrange todas as pessoas $\mathrm{e}$ populações e modela nossa forma de envelhecer, pois influencia todos os outros fatores determinantes do envelhecimento ativo. Através do gênero, considera-se a adequação de várias opções políticas, bem 
como o efeito dessas sobre o bem-estar de homens e mulheres ${ }^{(11)}$.

De acordo com a Organização Mundial da Saúde (OMS), idosos que possuem casa própria e que residem em ambientes mais seguros estão sujeitos a serem mais independentes, o que resulta em uma melhor qualidade de vida ${ }^{(12)}$.

Os determinantes econômicos exercem um papel relevante sobre o envelhecimento ativo, e assim destacam-se alguns fatores como: renda, proteção social e escolaridade. A pesquisa realizada vem mostrando que é crescente o número de idosas com seus rendimentos (aposentadoria e pensões), a família fora dos índices mais baixos de pobreza. As aposentadorias desempenham um papel muito importante no rendimento dos idosos, o que é mais evidenciado com o avançar da idade.

O perfil sociodemográfico e a qualidade de vida das idosas frequentadoras de um centro de referência para idosas se igualou com a média brasileira e a superou em alguns perfis, o que nos faz relacionar com nível de atividade física dessa população e as políticas públicas direcionadas à terceira idade.

Em relação as comorbidades avaliadas as mais prevalentes foram $\mathrm{o}$ sobrepeso e a queda, fatores determinantes que retardam um envelhecimento saudável e ativo. Estudos analisaram que a implantação de Estratégia da Saúde da Família (ESF) pode ser uma política importante, pois proporciona educação em saúde como medida de prevenção os fatores de risco $^{(13)}$.

\section{A Associação Brasileira para o} Estudo da Obesidade e da Síndrome Metabólica - ABESO, o aumento da obesidade ocorre em populações mais pobres e com menor escolaridade, por provável baixo custo de alimentos de grande densidade energética, além de fatores como: diminuição de refeições realizadas em casa; aumento da "alimentação rápida" como em redes de fastfood e o maior tamanho das porções "normais", levando ao aumento do conteúdo calórico das refeições ${ }^{(14)}$.

O processo de envelhecimento é diferente de um indivíduo para o outro, e estas são geradas por diversos fatores como condições socioeconômicas, doenças crônicas, mudanças no aspecto biológico. Outros caracterizam como a diminuição das Atividades de Vida Diárias (AVD). Há também quem considera que é um período de vulnerabilidade e de alta dependência da família. Outros consideram que a velhice é o ponto elevado da sabedoria ${ }^{(15)}$.

No que diz respeito aos determinantes do envelhecimento ativo nas mulheres idosas, é observado que na população estuda existiam poucos determinantes que dificultavam esse envelhecimento, destacando com maior nível 
os problemas visuais. O envelhecimento, um dos principais desafios colocados às sociedades contemporâneas. Verifica-se uma "requalificação" discursiva do envelhecimento, associando-o a uma pluralidade terminológica que desconstrói a prevalência de estereótipos idealistas negativos que associam a velhice a dependência, falta de autonomia, doença, institucionalização e uma desconsideração da sua heterogeneidade (de género, por exemplo) (16).

Alguns pontos podem ser identificados na pesquisa em relação as dificuldades encontradas para um envelhecimento ativo, entre estas podemos citar o uso de tapetes soltos que causam quedas, como também a falta de corrimão no banheiro que ajuda no equilíbrio das idosas. É notório a importância que as idosas mantenham sempre uma alimentação saudável, prática de atividade física, desenvolvimento cognitivo e da memória. Tudo isso contribui para que as idosas tenham um envelhecimento ativo e livre de muitas comorbidade.

Os idosos percebem o envelhecimento comportamentos inerentes ao estilo de vida, com destaque para a alimentação saudável, práticas de atividade físicas não ser tabagista e nem etilista. Esses hábitos e comportamentos são fatores de proteção e auxiliam no controle de doenças crônicas não saudável como a adoção de hábitos e

transmissíveis (DCNT). Com os dados analisados da pesquisa é possível notar que as mulheres que adotam um envelhecimento ativo têm menos predisposição a desenvolver problemas relacionados com a saúde devido o envelhecimento ${ }^{(17)}$.

Assim como os aspectos pessoais são importantes, os determinantes comportamentais talvez sejam os mais significativos para um envelhecimento ativo, pois são eles que podem ser modificados em qualquer período e tais mudanças podem representar um ganho relevante. O envolvimento em atividades físicas adequadas, a alimentação saudável, a abstinência do fumo e do álcool e, também, o uso de medicamentos, sabidamente, podem prevenir doenças e o declínio funcional, além de aumentar a longevidade e a qualidade de vida do indivíduo. A prática de exercícios físicos, além de promover saúde, pode ter influência positiva sobre a autoestima dos idosos, um fator determinante para que eles tenham motivação para manter um comportamento ativo na comunidade em que vivem $^{(18)}$.

O envelhecimento saudável é o desenvolvimento e manutenção da capacidade funcional que permite a qualidade de vida na idade em estágio avançado, definição essa baseada no conceito de capacidade intrínseca que se refere a capacidade física e mental que um indivíduo pode se apoiar e a capacidade funcional que são as características https://doi.org/10.31011/reaid-2021-v.95-n.36-art.1085 Rev Enferm Atual In Derme v. 95, n. 36, 2021 e-021143 
relacionadas a saúde que faz o indivíduo faça o que valoriza com motivo. Para a promoção do envelhecimento saudável é preciso desenvolver sistemas que que possa garantir o acesso de forma holística adequando a sua necessidade ${ }^{(19)}$.

\section{CONCLUSÃO}

Sabemos que as pessoas envelhecem de formas diferentes e, com base nos dados encontrados no presente estudo, pode-se concluir que apesar de nem todas as idosas terem obtido uma avaliação positiva em cada domínio do instrumento de avaliação multidimensional do envelhecimento ativo, em uma visão geral apresentaram-se ativos. A maioria das idosas participavam ativamente das atividades realizadas no centro de convivência o que só foi possível porque tiveram um processo de envelhecimento ativo oportunizado por seus comportamentos e seu contexto social.

Verificou-se, também, que o instrumento de avaliação dos determinantes do envelhecimento ativo constituiu uma importante ferramenta de pesquisa, permitindo identificar os fatores que constituem o envelhecimento ativo da população idosa estudada. Ao utilizá-lo, pode-se perceber o bom desempenho dos sujeitos pesquisados com relação aos fatores determinantes, tais como: realização de atividades físicas adequadas, alimentação saudável entre outros. Tais resultados permitem dizer que o grupo de idosos estudado apresentou comportamentos bastante saudáveis, os quais, possivelmente, influenciaram na longevidade alcançada e na qualidade de vida.

\section{REFERÊNCIAS}

1. Mantovani EP, Lucca SR, Neri AL. Associações entre significados de velhice e bemestar subjetivo indicado por satisfação em idosos. Rev. bras. geriatr. gerontol [Internet]. 2016 [acesso em 3 maio 2018]; 19 (2). Disponível em: http://www.scielo.br/scielo.php?pid=S1809$98232016000200203 \&$ script $=$ sci_abstract\&tlng=p $\mathrm{t}$

2. Vicente FR, Santos SMD. Avaliação multidimensional dos determinantes do envelhecimento ativo em idosos de um município de Santa Catarina. Texto Contexto Enferm [Internet]. 2013 [acesso em 5 maio 2018]; 22 (2): 370-378. Disponível em: https://doi.org/10.1590/S010407072013000200013.

3. Confortin SC, Schneider IJC, Antes DL, Cembranel F, Ono LM, Marques LP et al . Condições de vida e saúde de idosos: resultados do estudo de coorte EpiFloripa Idoso. Epidemiol. Serv. Saúde [Internet]. 2017 [acesso em 20 mai 2018]; 26(2): 305-317. Disponível em: http://www.scielo.br/scielo.php?script=sci_arttext \&pid=S2237-96222017000200305\&lng=en.

4. Caldeira S, Merighi MAB, Muñoz LA, Jesus MCP, Domingos SRF, Oliveira DM. O enfermeiro e o cuidado à mulher idosa: abordagem da fenomenologia social. Rev. LatinoAm. Enfermagem [Internet]. 2012 [acesso em 20 mai 2018]; 20(5): 888-895. Disponível em: http://www.scielo.br/scielo.php?script=sci_arttext \&pid=S0104-11692012000500010\&lng=en.

5. Wichmann FMA, Couto AN, Areosa SVC, Mntañés MCM. Grupos de convivência como suporte ao idoso na melhoria da saúde. Rev. Bras. Geriatr. Gerontol [Internet].2013 [acesso em 18 mai 2018]; 16 (4): 821-832, Disponível em: http://www.scielo.br/scielo.php?script=sci_arttext \&Ing=en. 
6. Siquenique, S. Avaliação Multidimensional da Pessoa Idosa: análise crítica da literatura e proposta de protocolo: domínios e instrumentos de Avaliação [Dissertação de Mestrado] Instituto Politécnico de Setúbal, Setúbal, Portugal; 2015.

7. Silva GF, Moura MAV, Almeida MV de S, Sá SPC, Queiroz ABA. Influências do climatério para o envelhecimento na percepção de mulheres idosas: subsídios para a enfermagem. Rev. Eletr. Enf. [Internet].2016 [acesso em 10 jun de 2018]; 17(3). Disponível em: https://www.fen.ufg.br/revista/v17/n3/pdf/v17n3a 09.pdf

8. Gil AC. Métodos e técnicas de pesquisa social. 6. ed. Editora Atlas SA, 2008.

9. Polit DF, Beck CT. Fundamentos de Pesquisa em Enfermagem: Avaliação de Evidências para a Prática da Enfermagem. Artmed Editora, 2016.

10. Brasil. Diretrizes e normas regulamentadoras da pesquisa envolvendo seres humanos. Brasil [Internet]. 2012 [acesso em 05 mai de 2018]. Disponível em: https://bvsms.saude.gov.br/bvs/saudelegis/cns/201 3/res0466_12_12_2012.html

11. Organização Pan - Americana de Saúde. Envelhecimento ativo: uma política de saúde/World Health Organization. Brasília (DF): Organização Pan-Americana de Saúde; 2005.

12. Brasil. Instituto Brasileiro de Geografia e Estatística (IBGE). Síntese de indicadores sociais [Internet]. 2016 [acesso em: 30 jun. 2019]; Disponível em:

https://www.ibge.gov.br/estatisticasnovoportal/sociais/educacao/9221-sintese-deindicadores-sociais.html?=\&t=downloads

13. Varela FRA, Ciconelli RM, Campolina AG, Soarez PC de. Quality of life evaluation of frail elderly in Campinas, São Paulo. Revista da Associação Médica Brasileira. [Internet]. 2015 [acesso em: 30 jul.2019]; 61(5). Disponível em: http://dx.doi.org/10.1590/1806-9282.61.05.423.

14. Abeso. Associação Brasileira para o Estudo da Obesidade e da Síndrome Metabólica. Diretrizes brasileiras de obesidade. 4ed. São Paulo: ABESO; $2016 . \quad$ Disponível em: http://www.abeso.org.br/uploads/downloads/92/5 7fccc403e5da.pdf

15. Fechine BRA, Trompieri N. O processo de envelhecimento: as principais alterações que acontecem com o idoso com o passar dos anos. InterSciencePlace [Internet]. 2015 [acesso em: 1 jun 2018]; 1(20).
16. Daniel F, Caetano E, Monteiro R, Amaral I. Representações sociais do envelhecimento ativo num olhar genderizado. Aná. Psicológica [Internet]. 2016 [acesso em: 20 jun 2018]; 34 (4). Disponível

em: http://dx.doi.org/10.14417/ap.1020.

17. Tavares RE, Jesus MCP de, Machado DR, Braga VAS, Tocantins FM, Merighi MAB. Envelhecimento saudável na perspectiva de idosos: uma revisão integrativa. Rev. Bras. Geriatr. Gerontol [Internet]. 2017. [acesso em: 01 agos 2018]; 20 (6): 889-900. Disponível em: http://doi.org/10.1590/1981-22562017020.170091

18. Organização mundial da saúde (OMS). Resumo: relatório mundial de envelhecimento. Rio de Janeiro: OMS; 2015 [acesso em 13 set 2017]; Disponível em: http://sbgg.org.br/wpcontent/uploads/2015/10/OMSENVELHECIMENTO-2015-port.pdf

19. Meurer ST, Benedetti TRB, Mazo GZ. Teoria da autodeterminação: compreensão dos fatores motivacionais e autoestima de idosos praticantes de exercícios físicos. Rev Bras Ativ Fis Saúde. 2011Fev; 16(1):18-24.

Submissão: 2021-04-16

Aprovado: 2021-06-22 